\title{
Neuromuscular electrical stimulation improves strength, pain and weight distribution on patients with knee instability post surgery
}

\author{
Yasuyoshi Asakawa ${ }^{\mathrm{a}}$, Ji-Hye Jung ${ }^{\mathrm{b}}$, Si-Eun $\mathrm{Koh}^{\mathrm{c}}$ \\ ${ }^{a}$ Graduate School of Health Sciences, Guma University, Gunma, Japan \\ ${ }^{\mathrm{b}}$ Physical Therapy Team, Hangeoleum Rehabilitation Hospital, Seoul, Republic of Korea \\ ${ }^{\mathrm{c}}$ Physical Therapy Team, Ilsan High Hospital, Goyang, Republic of Korea
}

Objective: The purpose of this study was to investigate the effects of an exercise with and without neuromuscular electrical stimulation (NMES) of the quadriceps femoris muscle, on strength, pain, and weight distribution in patients with knee instability post surgery.

Design: Randomized controlled trial.

Methods: Twenty patients in the early stage of rehabilitation after knee surgery were recruited as subjects and were randomly divided into either experimental group (exercise combined with NMES) $(n=10)$ or control group ( $n=10)$. Both groups received strength training of the lower limb for $20 \mathrm{~min} /$ day, 5 days/week for 4 weeks. The experimental group used NMES for unilateral quadriceps femoris training with incremental increases in the intensity of isometric contraction over 4 weeks. Outcome measurements were assessed using the digital manual muscle testing, 30-chair stand test (30CST), numeric pain rating scale (NPRS) and weight distribution using the foot analyzer before and after 4 weeks of training.

Results: After the 4-week intervention, knee extensor strength increased significantly in the experimental group post intervention $(p<0.05)$, and there was a significant improvement in the experimental group compared with the control group ( $p<0.05)$. The 30CST and NPRS scores improved significantly in the experimental group compared to the control group $(p<0.05)$, and there was a significant difference between the two groups $(p<0.05)$. Weight distribution was significantly improved in the experimental group compared with the control group, $(p<0.05)$, but there was no significant difference in improvement between the two groups. Conclusions: This study showed that NMES combined with strengthening exercises of the lower limbs is effective in improving lower limb pain and strength in patients with instability after knee surgery.

Key Words: Electrical stimulation, Muscle, Quadriceps muscle

\section{Introduction}

After knee surgery, weakness, pain and reduction of function of the quadriceps generally appear [1]. Asymmetric lower limb muscle strength due to surgery brings about instability of the knee joint [2]. Accordingly, asymmetry of weight distribution makes it difficult to perform daily life activities, such as walking and sit to stand $[1,3]$. In particular, quadriceps strength deficits of $50 \%-60 \%$ exist one month postoperatively [4]. Therefore, rehabilitation to secure muscle strength of the quadriceps is essential for function and stability of the knee [5].

Recently, neuromuscular electrical stimulation (NMES) has been used as an adjunct to traditional strengthening programs in patients post knee surgery. It has been suggested that NMES may provide a more effective means of increasing muscle strength than traditional strengthening programs [6]. NMES is the application of an electrical current to the

Received: 23 October, 2014 Revised: 25 November, 2014 Accepted: 30 November, 2014

Corresponding author: Si-Eun Koh

Physical Therapy Team, Ilsan High Hospital, 761 Goyang-daero, Ilsandong-gu, Goyang 410-315, Republic of Korea

Tel: 82-31-930-9894 Fax: 82-31-976-8345 E-mail: white3501@hotmail.com

(c) This is an Open-Access article distributed under the terms of the Creative Commons Attribution Non-Commercial License (http://creativecommons.org/licens es/by-nc/3.0) which permits unrestricted non-commercial use, distribution, and reproduction in any medium, provided the original work is properly cited.

Copyright @ 2014 Korean Academy of Physical Therapy Rehabilitation Science 
neuromuscular junction and the surrounding muscle fibers to cause a muscle contraction [7]. It can increase the muscle strength by increasing the load on the muscle using an electrically induced contraction to cause a training effect. Also it may provide a more effective means of mitigating quadriceps muscle central activation deficits and increasing quadriceps muscle strength than voluntary exercise alone [8]. Voluntary exercises may be limited after surgery because patients have difficulty producing sufficient muscle contraction, but NMES has the potential to override voluntary activation and can assist to reeducate the quadriceps to contract normally [9]. Talbot et al. [10] reported that after he carried out NMES to the quadriceps for 12 weeks on osteoarthritic patients, their muscle strength increased. These positive effects can be used for reduction of knee pain and strengthening of the quadriceps within the clinic, and can be applied with ease for patients who received surgery. Therefore, better resolution of activation deficits within the first month after surgery may offer a more effective rehabilitation strategy to improve recovery of quadriceps muscle function [11].

This study was conducted to investigate how much NMES affects the increase of muscle strength, reduction of pain, and weight distribution.

\section{Methods}

\section{Subjects}

This study selected 20 (6 men and 12 women) patients who had a total knee arthroplasty and due to being diagnosed with osteoarthritis and meniscus damage severe enough to necessitate surgery. They were divided into an experimental group composed of 10 participants who participated in a 4-week phased rehabilitation program and a control group of

Table 1. Standard postoperative rehabilitation program

\begin{tabular}{cc}
\hline Phase & Standard postoperative rehabilitation program \\
\hline 1 & Isometric strengthening of the quadriceps femoris \\
muscle in the form maximal isometric contractions \\
for 10 seconds each contraction was followed by \\
20 minutes \\
2 Straight leg raising exercise from crook lying \\
position for 45 limb elevation hold for 10 seconds, \\
then relax for 10 seconds, \\
3 Straight leg raising exercise from sitting position for \\
90 knee extension hold for 10 seconds, then relax \\
for 10 seconds, \\
4
\end{tabular}

10 participants. Between August 2014 and November 2014, all patients had undergone knee arthroscopy and total knee arthroplasty. Participants were excluded if they had inflammatory arthritis, implanted pacemakers or defibrillators, and dermatological conditions affecting the thigh. Prior to participation, subjects received an explanation of the objective and methods of the study and completed a consent form of agreement. The study protocol was approved by the institutional review board of Sahmyook University in Seoul, Korea.

\section{Procedures}

A garment-based NMES stimulator (Kneehab; BioMedical Research Ltd., Galway, Ireland) was used for the intervention. The device provided a stimulation frequency of $50 \mathrm{~Hz}$ and had an output current in the range 0 to $70 \mathrm{~mA}$. The Kneehab devices had a on/off ratio that was the same (1:1). Electrical stimulation parameters ramp-up time is 1 second and ramp-down time of 0.5 seconds was applied. Patients were instructed on the use of the NMES devices

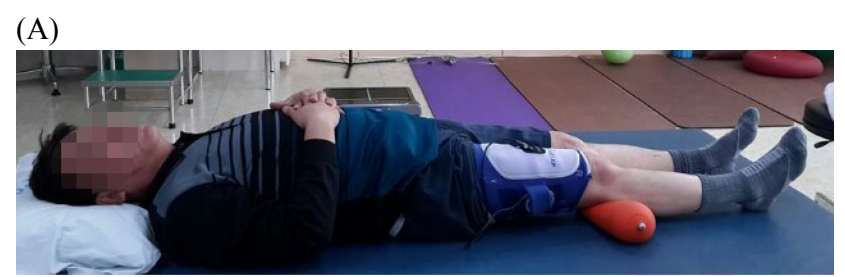

(B)

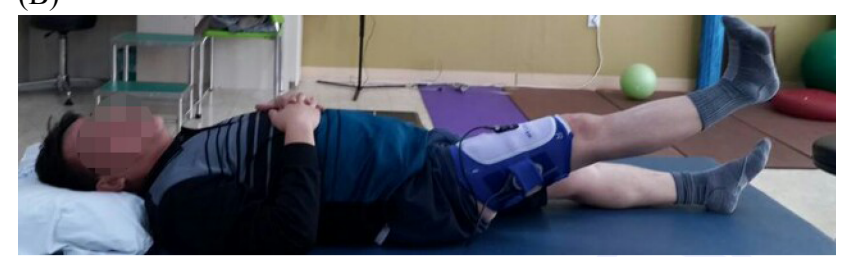

(C)

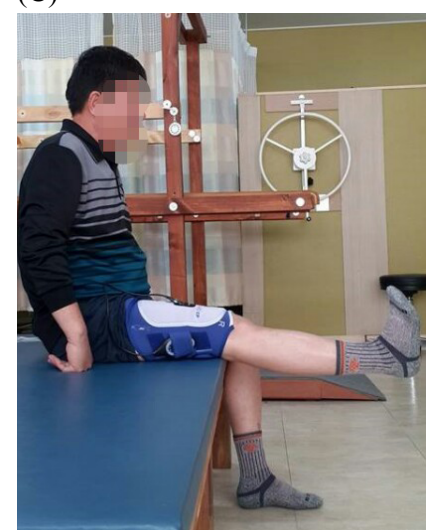

(D)

Figure 1. (A) Intervention 1 week, (B) intervention 2 week, (C) intervention 3 week, (D) intervention 4 week. 
during their stay in the hospital. For this research, muscle strength to participants was explained before starting the test and patients' consent was obtained after the providing the research purpose. The experimental group received a standard postoperative rehabilitation program combined with NMES, and the control group received only the standard postoperative rehabilitation program after a general surgery. They attended the program for 20 minutes a day, 5 times a week, for 4 weeks. Standard postoperative rehab program is as follows (Table 1, Figure 1). The protocol included straight leg raises, knee extensions with and without weights, continuous progressive movement, and flexibility exercises [12]. In both groups, training intensity and knee load gradually increased each week. Training intensity and amount of attention from the physical therapists were intended to be similar in both groups. Both groups were treated with the standard protocol by the same therapist.

\section{Outcome measures}

\section{Manual muscle testing}

The lower limb muscle strength was assessed by measuring the knee extensor using the Digital manual muscle testing (MMT) (Commander Muscle Tester; Jtechmedical, Midvale, ID, USA). MMT is of practical value as it is performed quickly without any equipment. The way of measuring muscular strength of lower limbs is to let a subject sit upright on chair that the subject's feet are about $20 \mathrm{~cm}$ from the ground and put the subject' hands on the thigh to measure the knee extensor. Muscle strength of the knee extensor was measured by giving resistance in the opposite direction of the subject's motion direction by attaching a dynamometer strip to the center part of the lower leg to be measured, and it was measured 3 times in total and the average value was recorded [13].

\section{0-chair stand test}

This test used 30-chair stand test (30CST) to estimate muscle strength of patients' lower limbs. This test decided it as once that examinees sat on a 17 inch-high chair with arms crossed over the chest and stood completely and then returned to a sitting position, which was measured by a stopwatch for 30 seconds and the number of times was recorded, which was measured a total of 3 times and it recorded the average value [14].

\section{Numeric pain rating scale}

The 11-point numeric pain rating scale (NPRS) was used to capture the patient's level of pain. The scale is anchored on the left with the phrase "no pain" and on the right with the phrase "worst imaginable pain." Examinees marked the position to assess their own pain levels ranging from " 0 " indicating no pain, to " 10 " indicating severe pain under each item of the questionnaire. The test-retest have been shown to be high reliability $(\mathrm{r}=0.95)$ [15].

\section{Weight distribution}

This research used a plantar foot pressure analysis (Foot Checker; GHiWell, Korea) to estimate the pressure distribution of static plantar foot pressure and surface area. The whole size of the Foot Checker is $520 \times 650 \mathrm{~mm}$, thickness of the pressure sensor is $0.15 \mathrm{~mm}$, the size of each sensor is $0.73 \mathrm{~cm}^{2}$, the number of the sensors equate to $2,304(48 \times 48)$, and voltage of $220 \mathrm{~V}, 50 / 60 \mathrm{~Hz}$. The plantar foot pressure was measured in a state where the subjects put their feet shoulder width apart on the sensor with their eyes toward the front, and the position was held for 10 seconds.

\section{Data analysis}

In this study, PASW Statistics ver. 18.0 (IBM Co., Armonk, NY, USA) was used for statistical analysis. The general characteristics of subjects were analyzed by using descriptive statistics (Table 2). A paired t-test was used to investigate the differences between the experimental group and the control group. The independent t-test was used to

Table 2. General characteristics of subjects

$(\mathrm{N}=20)$

\begin{tabular}{lccc}
\hline & NMES group $(\mathrm{n}=10)$ & Control group $(\mathrm{n}=10)$ & $X^{2} / t(p)$ \\
\hline Gender (male/female) & $2 / 8$ & $4 / 6$ & $-0.949(0.355)$ \\
Age $(\mathrm{y})$ & $45.50(12.376), 47.00(19-60)$ & $57.00(16.310), 54.00(20-76)$ & $1.776(0.093)$ \\
Height $(\mathrm{cm})$ & $163.00(7.409)$ & $160.50(12.607)$ & $0.541(-0.595)$ \\
Weight $(\mathrm{kg})$ & $67.30(9.093)$ & $64.60(10.469)$ & $-0.661(0.517)$ \\
\hline
\end{tabular}

Values are expressed as mean (SD), median (range) or mean (SD) only.

NMES: neuromuscular electrical stimulation. 
Table 3. Comparison of outcome variables lower extremity within group

$(\mathrm{N}=20)$

\begin{tabular}{lcc}
\hline \multicolumn{1}{c}{ Variable } & NMES group $(\mathrm{n}=10)$ & Control group $(\mathrm{n}=10)$ \\
\hline Mamual muscle test $(\mathrm{N})$ & & $91.900(11.4054)$ \\
Pre & $87.930(8.6225)$ & $96.49(17.437)$ \\
Post & $114.10(19.714)$ & 0.420 \\
$p$ & 0.001 & $4.40(1.713), 4.00(2-7)$ \\
Numeric pain rating scale (score) & & $3.10(1.524), 3.00(1-6)$ \\
Pre & $5.70(1.418), 5.50(4-8)$ & 0.022 \\
Post & $2.30(1.059), 2.00(1-5)$ & $9.50(2.838)$ \\
$p$ & 0.000 & $11.00(2.867)$ \\
30 second chair standing test (count) & $8.20(1.317)$ & 0.018 \\
Pre & $12.00(2.494)$ & $45.5390(1.88379)$ \\
Post & 0.000 & $48.1520(1.91725)$ \\
$p$ & & 0.006 \\
Weight distribution (\%)
\end{tabular}

Values are expressed as mean (SD) only or mean (SD), median (range).

compare the differences between the experimental group and the control. $p$-values $<0.05$ were considered significant.

\section{Results}

After the 4-week intervention, MMT scores were significantly improved in the experimental group $(p<0.05)$, compared to the control group $(p<0.05)$. As for the NPRS pain levels were significantly decreased in both groups post intervention $(p<0.05)$, In addition, the experimental group showed a more significant improvement compared to the control group. $(p<0.05)$. For the 30CTS, the experimental group showed a more significant level of improvement $(p$ $<0.05$ ), compared to the control group $(p<0.05)$. For weight distribution, both groups showed a significant increase post intervention $(p<0.05)$, but there was a no significant difference between both groups (Table 3, Figure 2).

\section{Discussion}

NMES offers an effective approach for mitigating quadriceps muscle central activation deficits early after surgery and restores normal quadriceps muscle function more effectively than voluntary exercise alone [16-18]. This study included an exercise to promote lower limb muscle strength, in addition to applying NMES to patients with knee joint instability due to residual weakness of the quadriceps after surgery. The study showed that the experimental group with NMES showed a more significant amount of improvement in knee extensor strength according to MMT scores, compared with the control group. It is considered that the combination of voluntary contraction training of the quadriceps and NMES caused a larger contraction of the muscle and promoted improvement of muscle strength. These results were identical with previous studies that reported that rehabilitation with combined voluntary contraction with NMES was better than training only with NMES or with voluntary contraction [16-18]. McAlindon et al. [19] predicted that weakness of quadriceps weakness would cause pain in the knee joint and the pain would restrict lower limb function. Also, quadriceps weakness has been consistently associated with knee pain thus affecting other joint movements [20-23]. This study also showed that according to the NPRS, a significant reduction of pain levels were indicated in both groups and that the difference between both groups was significant $(p<0.05)$. It is considered that exercise combined with NMES promoted rapid improvement of muscle strength. Knee pain results from factors that may be biological or psycho-social in origin. Among biological factors, data suggests that quadriceps weakness may contribute to worsened knee pain [24]. There are many examinations of function used for proving the protocol effect $[25,26]$, but it is time-consuming or there are not many special examinations focused on functional failure related with the quadriceps. This research included the 30CST to identify functional failure due to the weakening of the quadriceps. Mizner et al. [27] reported that muscle strength of the quadriceps after surgery was more strongly related with functional outcomes 

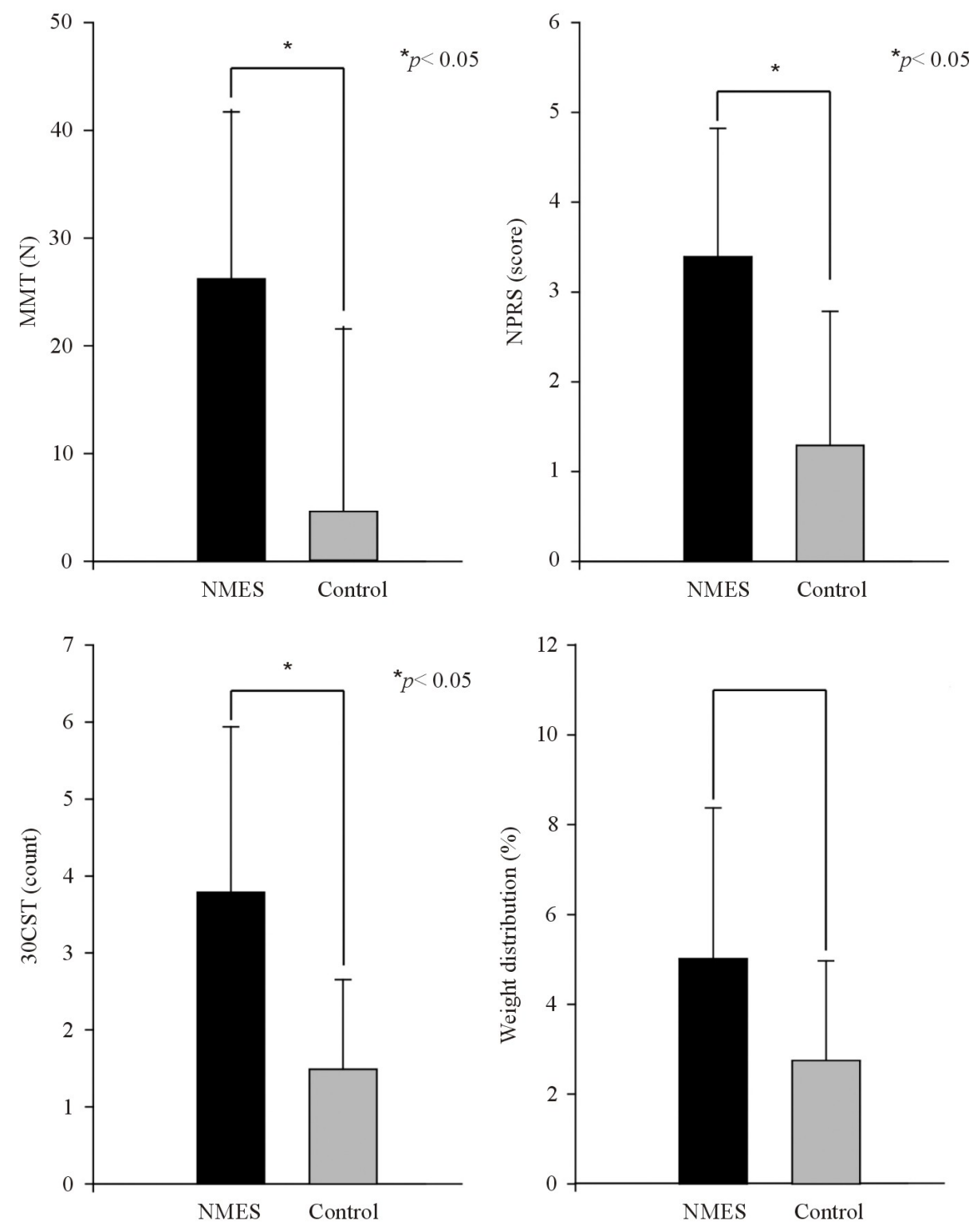

Figure 2. Changes between pre- and post-test of manual muscle test (MMT), numeric pain rating scale (NPRS), 30 scond chair test (30CST), weight distribution. " Statistically significant difference compared to control group $(p<0.05)$.

rather than flexion range of the knee joint. In a previous study, the group with NMES applied demonstrated faster rising from the chair, pain reduced and increased knee extensor strength [10]. The study showed that there was a significant difference in level of improvement in both groups and that there was also a significant increase in values of before and after intervention between both groups $(p<0.05)$. It is considered that the muscle strengthening training of the quadriceps applied to both groups after surgery led to improvement of muscle strength and functional performance. The 30CST was used to observe functional mobility and was highly correlated with pain and weight distribution [14,28,29]. Asay et al. [30] showed that knee joint pain brought about asymmetry of motion, including weight support of both lower limbs. In this study, both groups showed a significant increase in the ratio of weight support $(p<0.05)$. This result indicate that the recovery of muscle strength and pain was related with weight loaded on both lower limbs after training The research results seen based on the precedent study has shown that pain of both groups improved significantly and affected weight distribution of both lower limbs. However, the general rehabilitation program combined with NMES only concentrated on the quadriceps. However, weight distribution of both lower limbs in standing position does not simply use the muscular strength of the quadriceps but lower limb coordination is also considered as 
an important factor [31]. In addition, as motions requiring muscle strength of both lower limbs among the general rehab programs after the surgery applied by this study are also included, it is considered that weight distribution in both groups would increase significantly. As a result of this study, the improvement of quadriceps muscle in the rehabilitation after the knee joint surgery had a great influence on the function of lower limbs. For that reason, stability of the lower muscles influenced symmetric distribution of weight. Our results suggest that rehabilitation protocols that promote muscle strengthening using only voluntary exercise may be insufficient to address quadriceps weakness after knee surgery because failure of voluntary activation is a significant contributor to the loss of quadriceps strength after surgery. Eventually, this is considered that an auxiliary role of NMES in the early stage of rehabilitation became a necessary role so that it can access the voluntary exercise, the last stage of rehabilitation, more easily. However, NMES does not promote the complicated exercise control. Not only is the agonist important but also coordination with an antagonist is an important element for complicated movements [8]. Therefore, it is considered that the coordination ability with a different antagonist is a necessity to the rehabilitation program. There were several limitations to the present study that should be considered. First, this study included a small sample size. Second, personal surgery types or levels of physical activity were not equivalent. Third, there is a necessity for follow-up and observation to identify how the effect continues after leaving hospital. It is suggested that further studies are conducted for further comparison of the effects of NMES according to the disease and condition of the knee joint.

\section{Conflict of Interest}

The authors declared no potential conflicts of interest with respect to the authorship and/or publication of this article.

\section{References}

1. Stevens JE, Mizner RL, Snyder-Mackler L. Quadriceps strength and volitional activation before and after total knee arthroplasty for osteoarthritis. J Orthop Res 2003;21:775-9.

2. Petterson SC, Barrance P, Buchanan T, Binder-Macleod S, Snyder-Mackler L. Mechanisms underlying quadriceps weakness in knee osteoarthritis. Med Sci Sports Exerc 2008;40:422-7.

3. Mizner RL, Snyder-Mackler L. Altered loading during walking and sit-to-stand is affected by quadriceps weakness after total knee arthroplasty. J Orthop Res 2005;23:1083-90.

4. Stevens-Lapsley JE, Balter JE, Kohrt WM, Eckhoff DG. Quadriceps and hamstrings muscle dysfunction after total knee arthroplasty. Clin Orthop Relat Res 2010;468:2460-8.

5. Frost H, Lamb SE, Robertson S. A randomized controlled trial of exercise to improve mobility and function after elective knee arthroplasty. Feasibility, results and methodological difficulties. Clin Rehabil 2002;16:200-9.

6. Lewek M, Stevens J, Snyder-Mackler L. The use of electrical stimulation to increase quadriceps femoris muscle force in an elderly patient following a total knee arthroplasty. Phys Ther 2001;81:1565-71.

7. Petterson S, Snyder-Mackler L. The use of neuromuscular electrical stimulation to improve activation deficits in a patient with chronic quadriceps strength impairments following total knee arthroplasty. J Orthop Sports Phys Ther 2006;36:678-85.

8. Paillard T. Combined application of neuromuscular electrical stimulation and voluntary muscular contractions. Sports Med 2008;38:161-77.

9. Doucet BM, Lam A, Griffin L. Neuromuscular electrical stimulation for skeletal muscle function. Yale J Biol Med 2012;85: 201-15.

10. Talbot LA, Gaines JM, Huynh TN, Metter EJ. A home-based pedometer-driven walking program to increase physical activity in older adults with osteoarthritis of the knee: a preliminary study. J Am Geriatr Soc 2003;51:387-92.

11. Thomas AC, Stevens-Lapsley JE. Importance of attenuating quadriceps activation deficits after total knee arthroplasty. Exerc Sport Sci Rev 2012;40:95-101.

12. Topp R, Swank AM, Quesada PM, Nyland J, Malkani A. The effect of prehabilitation exercise on strength and functioning after total knee arthroplasty. PM R 2009;1:729-35.

13. Li RC, Jasiewicz JM, Middleton J, Condie P, Barriskill A, Hebnes $\mathrm{H}$, et al. The development, validity, and reliability of a manual muscle testing device with integrated limb position sensors. Arch Phys Med Rehabil 2006;87:411-7.

14. Jones CJ, Rikli RE, Beam WC. A 30-s chair-stand test as a measure of lower body strength in community-residing older adults. Res Q Exerc Sport 1999;70:113-9.

15. Jensen MP, Turner JA, Romano JM. What is the maximum number of levels needed in pain intensity measurement? Pain 1994;58:387-92.

16. Stevens JE, Mizner RL, Snyder-Mackler L. Neuromuscular electrical stimulation for quadriceps muscle strengthening after bilateral total knee arthroplasty: a case series. J Orthop Sports Phys Ther 2004;34:21-9.

17. Avramidis K, Karachalios T, Popotonasios K, Sacorafas D, Papathanasiades AA, Malizos KN. Does electric stimulation of the vastus medialis muscle influence rehabilitation after total knee replacement? Orthopedics 2011;34:175.

18. Stevens-Lapsley JE, Balter JE, Wolfe P, Eckhoff DG, Kohrt WM. Early neuromuscular electrical stimulation to improve quadriceps muscle strength after total knee arthroplasty: a randomized controlled trial. Phys Ther 2012;92:210-26.

19. McAlindon TE, Cooper C, Kirwan JR, Dieppe PA. Determinants of disability in osteoarthritis of the knee. Ann Rheum Dis 1993;52:258-62.

20. O'Reilly SC, Jones A, Muir KR, Doherty M. Quadriceps weak- 
ness in knee osteoarthritis: the effect on pain and disability. Ann Rheum Dis 1998;57:588-94.

21. Hall MC, Mockett SP, Doherty M. Relative impact of radiographic osteoarthritis and pain on quadriceps strength, proprioception, static postural sway and lower limb function. Ann Rheum Dis 2006;65:865-70.

22. Madsen OR, Bliddal H, Egsmose C, Sylvest J. Isometric and isokinetic quadriceps strength in gonarthrosis; inter-relations between quadriceps strength, walking ability, radiology, subchondral bone density and pain. Clin Rheumatol 1995;14:308-14.

23. Jung JH, Ko SE, Lee SW. Immediate effects of single-leg stance exercise on dynamic balance, weight bearing and gait cycle in stroke patients. Phys Ther Rehabil Sci 2014;3:49-54.

24. Glass NA, Torner JC, Frey Law LA, Wang K, Yang T, Nevitt MC, et al. The relationship between quadriceps muscle weakness and worsening of knee pain in the MOST cohort: a 5-year longitudinal study. Osteoarthritis Cartilage 2013;21:1154-9.

25. Stratford PW, Kennedy DM, Woodhouse LJ. Performance measures provide assessments of pain and function in people with advanced osteoarthritis of the hip or knee. Phys Ther 2006;86: 1489-96.
26. Wright AA, Cook CE, Baxter GD, Dockerty JD, Abbott JH. A comparison of 3 methodological approaches to defining major clinically important improvement of 4 performance measures in patients with hip osteoarthritis. J Orthop Sports Phys Ther 2011;41:319-27.

27. Mizner RL, Petterson SC, Snyder-Mackler L. Quadriceps strength and the time course of functional recovery after total knee arthroplasty. J Orthop Sports Phys Ther 2005;35:424-36.

28. Boonstra MC, Schwering PJ, De Waal Malefijt MC, Verdonschot N. Sit-to-stand movement as a performance-based measure for patients with total knee arthroplasty. Phys Ther 2010;90:149-56.

29. Parent E, Moffet H. Preoperative predictors of locomotor ability two months after total knee arthroplasty for severe osteoarthritis. Arthritis Rheum 2003;49:36-50.

30. Asay JL, Mündermann A, Andriacchi TP. Adaptive patterns of movement during stair climbing in patients with knee osteoarthritis. J Orthop Res 2009;27:325-9.

31. Harato K, Nagura T, Matsumoto H, Otani T, Toyama Y, Suda Y. Extension limitation in standing affects weight-bearing asymmetry after unilateral total knee arthroplasty. J Arthroplasty 2010;25:225-9. 